\title{
Photoresponsive Polymers. Photostimulated Viscosity Change of Poly(3-vinyl-10-methylphenothiazine) in 1,2-Dichloroethane in the Presence of Carbon Tetrachloride
}

\author{
Dawan Kungwatchakun, Koichiro HaYaShI, and Masahiro IRIE \\ The Institute of Scientific and Industrial Research, \\ Osaka University, Ibaraki, Osaka 567, Japan
}

(Received November 21, 1987)

\begin{abstract}
The solution viscosity of poly(3-vinyl-10-methylphenothiazine) in 1,2-dichloroethane was found to decrease by as much as $50 \%$ on exposure to ultraviolet light $(\lambda>290 \mathrm{~nm})$ in the presence of carbon tetrachloride. The reduced viscosity again returned to the initial value by the addition of reductants, such as sodium dithionite. Absorption and ESR measurements of the solution suggested the formation of radical cations of pendant methylphenothiazine (MPT) groups by the interaction of the photoexcited MPT with carbon tetrachloride. Aggregation of ion-pairs of the cations and chloride ions due to dipole-dipole interactions is considered to be the driving force of the chain contraction. The addition of sodium dithionite reduces the radical cations to neutral forms and the chain again returns to the initial random conformation.
\end{abstract}

KEY WORDS Photoresponsive Polymers / Poly(3-vinyl-10methylphenothiazine) / Radical Cation / Photoviscosity Effect /

By incorporating a small amount of photoionizable chromophores into polymers, we have shown that the polymer chain conformation changes reversibly by photoirradiation. ${ }^{1}$ Poly $(N, N$-dimethylacrylamide) having pendant triphenylmethane leucohydroxide groups expands the conformation on exposure to ultraviolet light. The expanded conformation returns to the initial random conformation in the dark. The leucohydroxide chromophores dissociate into unstable ionpairs upon ultraviolet irradiation with production of colored triphenylmethyl cations along the polymer chain and in the dark the cations recombine with the counter ions.

To produce stable ions, which do not disappear in the dark, in pendant groups upon photoirradiation, we prepared poly(3-vinyl10-methylphenothiazine) (1, polyVMPT), and examined the photoresponsive behavior.

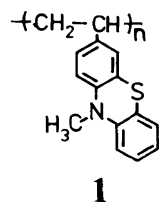

Phenothiazine and its derivatives are well known to undergo electrochemical oxidation to produce a radical cation in polar solvents. ${ }^{2}$ The electrochemical properties of polymers with pendant MPT groups have been extensively studied by Morishima et al. ${ }^{3}$

Photochemical oxidation of phenothiazine is reported to produce a neutral radical in ethanol by splitting off a hydrogen from nitrogen. ${ }^{2 a}$ When the mobile hydrogen is substituted by a methyl group, the MPT radical cation, instead of the neutral radical, is expected to be produced by photoirradiation in the presence of electron acceptors. 


\section{EXPERIMENTAL}

\section{Materials}

3-Vinyl-10-methylphenothiazine (VMPT) was synthesized according to the method of Gipstein. ${ }^{4}$ VMPT was isolated by column chromatography on silica and then purified by recrystallization from chloroform-methanol. Soft pale yellow needles were obtained (24 $\%$ yield). mp $94-96^{\circ} \mathrm{C}$. Anal. calcd for $\mathrm{C}_{15} \mathrm{H}_{13} \mathrm{~N}_{\mathrm{S}}$ : C, $75.28 \%$; H, 5.47\%; N, 5.85\%; S, $13.40 \%$. Found: C, $75.23 \%$;, $5.60 \%$; $5.77 \% ; \mathrm{S}, 13.35 \%$.

Solvents were distilled twice before use.

\section{Polymerization}

PolyVMPT was obtained by the radical polymerization of VMPT using AIBN as initiator in tetrahydrofuran (THF) at $60^{\circ} \mathrm{C}$. The polymer was purified by reprecipitation with dry acetone from THF solution. The molecular-weight of the polymer was estimated to be $3.2 \times 10^{4}$ with a gel-permeation chromatography (Toyo-Soda HLC-802) by comparison with standard polystyrenes.

\section{Measurements}

Photoirradiation was carried out with a Xe lamp (Ushio $15 \mathrm{w}$ ). The light passed through a cut-off filter (Toshiba UV29, $\lambda>290 \mathrm{~nm}$ ) was used. $\gamma$-Irradiation was carried out with ${ }^{60} \mathrm{Co}$ (dose rate $1 \mathrm{Mrad} / \mathrm{h}$ ). Conformational change of the polymer was followed by measuring the viscosity of the polymer solutions at $30^{\circ} \mathrm{C}$ with an Ubbelohde viscometer specially designed for irradiation. Absorption spectra were measured using a spectrophotometer (Shimadzu MPS-2000s).

\section{RESULTS AND DISCUSSION}

\section{Photoirradiation Effects on Viscosity}

Figure 1 shows the viscosity of polyVMPT in the dark as well as after ultraviolet irradiation for $20 \mathrm{~min}$ in a mixed solvent of carbon tetrachloride and 1,2-dichloroethane (volume

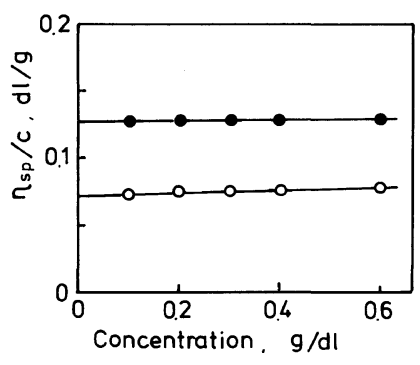

Figure 1. Viscosity of polyVMPT in a mixed solvent of carbon tetrachloride and 1,2-dichloroethane (volume ratio $=1: 4)$ at $30^{\circ} \mathrm{C}(O)$ in the dark and $(O)$ after ultraviolet irradiation $(\lambda>290 \mathrm{~nm})$ for $20 \mathrm{~min}$.

ratio $=1: 4)$. The intrinsic viscosity after ultraviolet irradiation was $45 \%$ lower than the viscosity in the dark. This indicates that the polymer shrinks upon ultraviolet irradiation. The possibility that the decrease is due to the degradation of the polymer chain is excluded, because the reduced viscosity again returned to the initial value by the addition of reductants, such as sodium dithionite, $\mathrm{Na}_{2} \mathrm{~S}_{2} \mathrm{O}_{4}$. Gel permeation chromatography measurement also indicated that the polymer degradation after ultaviolet irradiation is negligible.

Similar photo-decrease of the solution viscosity was observed in $o$-dichlorobenzene in the presence of carbon tetrachloride. In benzene or THF, precipitation of the polymer was observed after ultraviolet irradiation.

Figure 2 shows the dependence of the decrease of the solution viscosity on the photoirradiation time along with the increase of the absorption intensity at $525 \mathrm{~nm}$, attributable to the radical cation of the pendant MPT groups (see next section). The good correlation between the viscosity and absorption intensity changes indicates that the shrinkage of the polymer chain is due to the formation of positive charges along the polymer chain. By the addition of sodium dithionite, the red colors due to the radical cation disappeared and at the same time the viscosity returned to the initial value. This result also supports the mechanism that the formation of radical ca- 


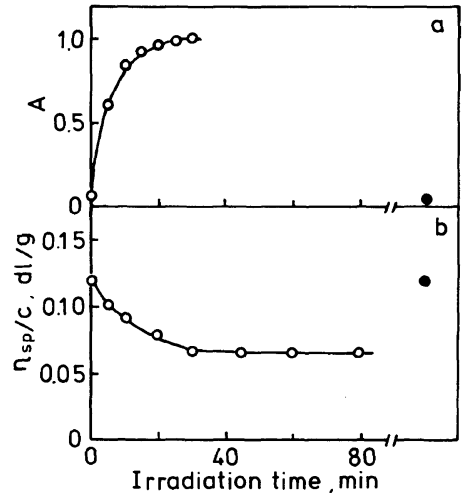

Figure 2. Changes of (a) the absorption intensity at $525 \mathrm{~nm}$ and (b) viscosity of polyVMPT in a mixed solvent of carbon tetrachloride and 1,2-dichloroethane (volume ratio $=1: 4$ ) upon ultraviolet irradiation $(\lambda>290 \mathrm{~nm})$ at $30^{\circ} \mathrm{C}$. (O) were measured after the addition of a droplet of methanol solution of sodium hydrosulfite. Concentration of the polymer is $0.1 \mathrm{~g} \mathrm{dl}^{-1}$. An absorption cell with $1 \mathrm{~mm}$ optical path length was used.

tion shrinks the polymer conformation.

The decrease of the solution viscosity depended on the polarity of the solvent. By changing the ratio of carbon tetrachloride (dielectric constant, 2.2) and 1,2-dichloroethane (10.4) in the mixed solvent, the solvent polarity was controlled. In less polar solvents, the ratio of the viscosity during ultraviolet irradiation to that in the dark decreased as shown in Figure 3. This suggests that the polymer with the ionized pendant groups has a compact form in less polar solvents. In the experiment, the concentrations of the radical ions in the pendant groups were adjusted to the same value.

The photostimulated viscosity decrease was not affected by the addition of salt in contrast with the result observed for $\operatorname{poly}(N, N$ dimethylacrylamide) with pendant triphenylmethane leucohydroxide groups. ${ }^{1}$ Even in the presence of $10^{-2} \mathrm{M}$ tetra- $n$-butylammonium perchlorate, the viscosity decreased by as much as $50 \%$ upon photoirradiation. This results clearly indicates that electrostatic force does not play an important role in the confor-

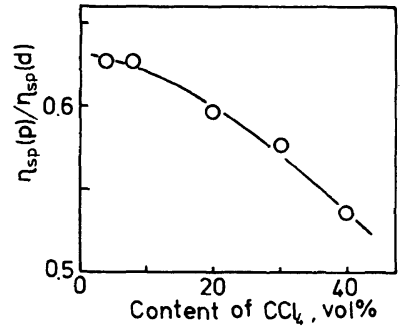

Figure 3. Solvent polarity dependence of the photodecrease of the solution viscosity of polyVMPT at $30^{\circ} \mathrm{C}$. Concentration of the polymer is $0.06 \mathrm{~g} \mathrm{dl}^{-1} \cdot \eta_{\mathrm{sp}}(p)$ and $\eta_{\mathrm{sp}}(d)$ are the specific viscosities under ultraviolet irradiation $(\lambda>290 \mathrm{~nm})$ and in the dark, respectively.

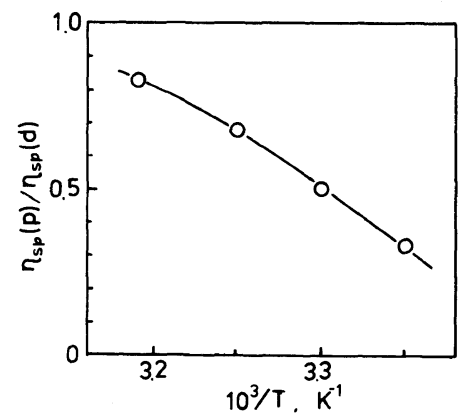

Figure 4. Temperature dependence of the photodecrease of the solution viscosity of polyVMPT in a mixed solvent of carbon tetrachloride and 1,2-dichloroethane (volume ratio $=1: 4$ ). Concentration of the polymer is $0.1 \mathrm{~g} \mathrm{dl}^{-1} \cdot \eta_{\mathrm{sp}}(p)$ and $\eta_{\mathrm{sp}}(d)$ are the specific viscosities under ultraviolet irradiation $(\lambda>290 \mathrm{~nm})$ and in the dark, respectively.

mational change of the present system.

Figure 4 shows the temperature dependence of the ratio of viscosity during ultraviolet irradiation to that in the dark. The ratio decreased at higher temperature. The reduced viscosity of $0.1 \mathrm{~g} \mathrm{dl}^{-1}$ polymer solution decreased by as much as $67 \%$ upon photoirradiation at $25^{\circ} \mathrm{C}$, while the photostimulated viscosity decrease was only $18 \%$ at $40^{\circ} \mathrm{C}$. The temperature dependence suggests that the contraction of the polymer chain is controlled by weak interactions.

\section{Spectroscopic Study}

Absorption spectral measurements give detailed information concerning the photore- 
actions in the system. Figure 5 shows the absorption spectrum of polyVMPT in the mixed solvent of carbon tetrachloride and 1,2dichloroethane (volume ratio $=1: 4$ ). Upon ultraviolet irradiation, absorption bands at 530 and $820 \mathrm{~nm}$ appeared. The bands were not observed in the absence of carbon tetrachloride.

In order to identify the bands, matrix isolation method combined with $\gamma$-irradiation technique was employed. According to Hamill, ${ }^{5}$ solute radical cation is produced by $\gamma$-irradiation in halogenated hydrocarbon matrices at $77 \mathrm{~K}$. Figure $6 \mathrm{a}$ and $\mathrm{b}$ show the absorption spectra of MPT and polyVMPT in $n$-butylchloride at $77 \mathrm{~K}$ after $\gamma$-irradiation, respectively. Upon $\gamma$-irradiation, a strong absorption band appeared at $525 \mathrm{~nm}$ and weak ones at 780 and $880 \mathrm{~nm}$ in the matrix containing MPT. These bands are ascribable to a MPT radical cation. ${ }^{6}$ When the sample was annealed at slightly higher temperature, the band at $525 \mathrm{~nm}$ showed a blue shift to $472 \mathrm{~nm}$ and a new broad absorption band appeared at $770 \mathrm{~nm}$. The band at 472 and $770 \mathrm{~nm}$ are ascribable to a dimer radical ion of MPT, produced by the raction of the radical cation with the neutral molecule. ${ }^{7,8}$ Figure $6 \mathrm{~b}$ shows the radical cation of polyVMPT, which has absorption bands at 540, 780, and $860 \mathrm{~nm}$. Upon annealing the sample at slightly higher temperature, the band at $540 \mathrm{~nm}$ showed a blue shift to $525 \mathrm{~nm}$ and a new band appeared at $820 \mathrm{~nm}$. Similar spectral change on annealing as observed in monomeric MPT suggests that the bands at 525 and $820 \mathrm{~nm}$ are assigned to a dimer radical cation, produced by the intramolecular interaction of a pendant MPT radical cation with a neighboring pendant group. Under the present experimental conditions, the ratio of ionized pendant groups to the neutral ones is estimated to be less than $10 \%$. Therefore, the assignment to a radical cation dimer ${ }^{3}$ cannot be adopted.

According to the above assignment, the spectrum observed in Figure 5 is ascribed to

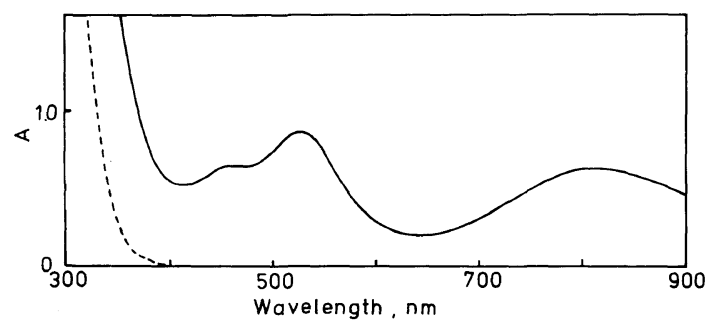

Figure 5. Absorption spectra of polyVMPT in a mixed solvent of carbon tetrachloride and 1,2-dichloroethane (volume ratio $=1: 4)(---)$ before photoirradiation and $(\longrightarrow)$ after ultraviolet irradiation $(\lambda>290 \mathrm{~nm})$ for $10 \mathrm{~min}$. An absorption cell with $1 \mathrm{~mm}$ optical path length was used.
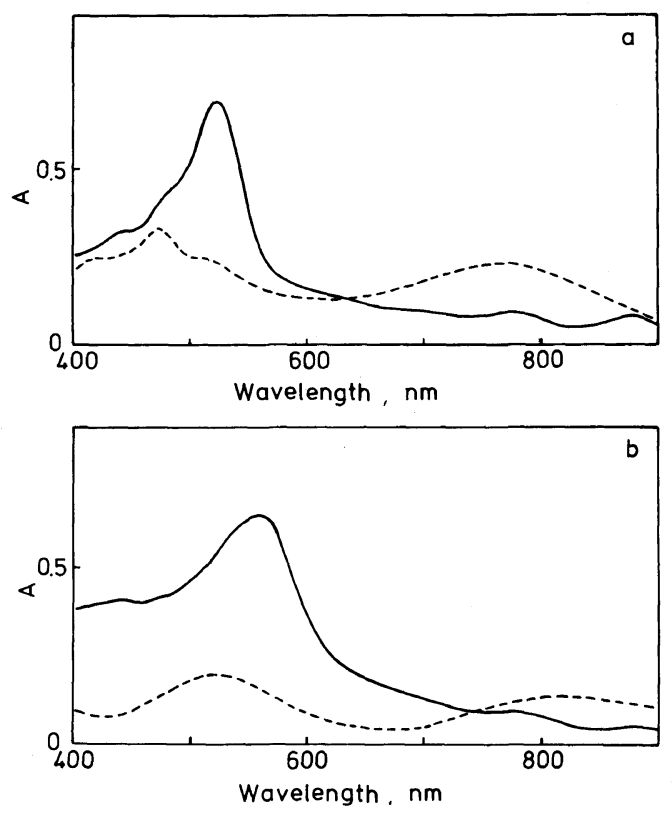

Figure 6. Absorption spectra of MPT in $n$ butylchloride at $77 \mathrm{~K} \mathrm{(-)} \mathrm{immediately} \mathrm{after} \gamma$-irradiation (1 Mrad) and (---) after annealing at slightly higher temperature. Concentration of MPT is $8 \times 10^{-3}$ moll $1^{-1}$. (b) Absorption spectra of polyVMPT in $n$ butylchloride at $77 \mathrm{~K}(-)$ immediately after $\gamma$-irradiation (1 Mrad) and (---) after annealing at slightly higher temperature. Concentration of the polymer is $0.8 \mathrm{~g} \mathrm{dl}^{-1}$.

the dimer radical cation of the pendant MPT groups. In order to confirm the assignment, ESR spectrum of the polymer was measured in the mixed solvent of carbon tetrachloride and 1,2-dichloroethane (volume ratio $=1: 4$ ). A 
very strong singlet with $\Delta H_{\mathrm{ms}}=1.5 \mathrm{mT}$ was detected. The ESR spectrum indicates that the photogenerated species has a paramagnetic property. The possibility of the absorption bands at 530 and $820 \mathrm{~nm}$ due to the dimer of the two radical cations is excluded in the present system, because the dimer is diamagnetic.

\section{Mechanism of Photostimulated Viscosity \\ Change}

Upon ultraviolet irradiation, polyVMPT in the mixed solvent of carbon tetrachloride and 1,2-dichloroethane gives visible absorption bands at 530 and $830 \mathrm{~nm}$ and a singlet ESR spectrum, which are assigned to dimer radical cations of pendant MPT. These spectra were not observed in the absence of carbon tetrachloride. The results indicate that photoexcited MPT pendant groups ionize by the interaction with carbon tetrachloride as follows,

$$
\begin{aligned}
\int-M P T & \stackrel{h \nu}{\rightarrow} \int-M P T^{*} \\
f M P T^{*}+\mathrm{CCl}_{L} & \rightarrow f-\mathrm{MPT}^{+}+\mathrm{Cl}^{-}+\cdot \mathrm{CCl}_{3}
\end{aligned}
$$

Then the monomer radical cations associate with neutral MPT molecules in the pendant groups to produce dimer radical cations as follows,

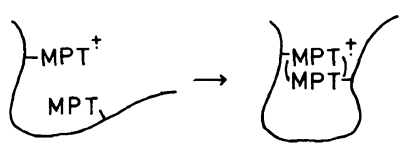

The formation of radical ions along the polymer chain should expand the polymer chain, if the electrostatic repulsive forces contributed to the conformational change. The polymer chain, on the contrary, contracted upon ultraviolet irradiation. In the solvents used in the present experiment, almost all ions are in pairs with the counter ions and the electrostatic repulsive forces play a minor role. This was confirmed by the fact that the addition of salts did not affect the photostimulated viscosity decrease. Instead, intramolecular aggregation of the ion-pairs due to dipoledipole interactions are considered to take place and the polymer chains contract upon photoirradiation. Although at the polymer concentration used intermolecular aggregation was not observed, the intermolecular interaction is expected to occur at higher concentration. Intramolecular dimer radical cation formation may also contribute to the contraction of the polymer chains to some extent.

Acknowledgements. We are grateful to Dr. Y. Morishima, Osaka University, for his valuable discussions and also helpful advice on the synthesis of VMPT.

\section{REFERENCES}

1. M. Irie and M. Hosoda, Makromol. Chem. Rapid Commun., 6, 533 (1985).

2. a) H. J. Shine and E. E. Mach, J. Org. Chem., 30, 2130 (1965); b) D. Clarke, B. C. Gilbert, P. Hanson, and C. M. Kirk, J. Chem. Soc., Perkin Trans. 2, 1103 (1978).

3. a) Y. Morishima, Y. Itoh, and K. Koyagi, J. Polym. Sci., Polym. Chem. Ed., 21, 953 (1983); b) Y. Morishima, I. Akihara, H. S. Lim, and S. Nozakura, Macromolecules, 20, 978 (1987).

4. E. Gipstein, W. A. Hewett, and O. U. Need, J. Polym. Sci., A-1, 8, 3285 (1970).

5. W. H. Hamill, "Radical Ions," E. T. Kaiser and L. Kevan, Eds., Interscience, New York, 1968.

6. H. J. Shin, D. R. Thompson, and C. Veneziani, $J$. Heterocycl. Chem., 4, 517 (1967).

7. B. Badger and B. Brocklehurst, Trans. Faraday Soc., 65, 2576, 2582, 2588 (1967).

8. S. Irie, H. Horii, and M. Irie, Macromolecules, 13, 1355 (1980). 